\title{
Incident Reporting System in Pediatric Intensive Care Units of Cairo Tertiary Hospital: An Intervention Study
}

\author{
John Rene Labib ${ }^{1}$, Nesreen Mohamed Kamal Elden (iD ${ }^{2,}{ }^{,}$, Amira Aly Hegazy ${ }^{2}$ and Nargis Albert Labib ${ }^{2}$ \\ ${ }^{1}$ Pediatrics Department, Faculty of Medicine, Cairo University, Cairo, Egypt \\ ${ }^{2}$ Public Health Department, Faculty of Medicine, Cairo University, Cairo, Egypt \\ "Corresponding author: Public Health Department, Faculty of Medicine, Cairo University, Cairo, Egypt. Email: drn_kamal@kasralainy.edu.eg
}

Received 2019 March 22; Revised 2019 September 08; Accepted 2019 October 28.

\begin{abstract}
Background: Incident reporting system (IRS) deepens the understanding of the frequency of adverse events and near misses. Voluntary reporting is an essential step to improve patient safety.

Objectives: The study aimed to apply an efficient and reliable system for incident reporting to enhance patient safety practices in pediatric intensive care units (PICUs).

Methods: A quasi-experimental pretest-posttest study design was conducted to implement a voluntary anonymous IRS in PICUs. In-depth interviews were conducted with 16 health care personnel. A tailored educational program was provided to 73 health care personnel. A questionnaire was administered before and three months after the intervention to assess their attitude towards incident reporting.

Results: The interviewed health care providers highlighted that no IRS was established in the PICUs and most of them never reported any event unless it was a sentinel event. They agreed that an IRS would be beneficial to PICUs. The average percentage of positive responses for "Frequency of error reporting' increased significantly from $23.8 \%$ to $42 \%$. Communication problems, hygienic errors, therapeutic errors, and diagnostic errors accounted for 34\%, 32\%, 29\%, and 5\% of the reported potential errors, respectively.

Conclusions: IRS implementation improves potential error reporting attitude and practice in PICUs.
\end{abstract}

Keywords: Reporting, Errors, PICU, Near Miss

\section{Background}

Preventable harm to patients due to their health care should never be acceptable. An adverse event was defined by Wilson et al. in 2008 as "an unintended injury caused by health care management rather than by the patient's underlying disease that results in temporary or permanent disability, death or prolonged hospital stay" (1). Although health care interventions are intended to help patients, they can also harm patients (2). The latest conservative estimates show that patient harm is now ranked the 14 th leading cause of morbidity and mortality in the world (3).

Patient safety is considered a major public health and human right issue. The rate of adverse events is at least $8 \%$ among in-patients at hospitals of the developed world and $0.5 \%-2 \%$ of patients' deaths at hospitals are associated with adverse events, which are often preventable (3, 4 ). However, a huge knowledge gap exists on the actual rates of adverse events in developing countries, which has been a serious limitation to understanding the extent of the problem in the global context. Up to 2012, the majority of published studies showed reports from developed countries. However, a large-scale retrospective medical record review study was conducted to estimate the type and frequency of adverse events in 26 hospitals of eight developing countries from the Eastern Mediterranean Sea and Africa (5). The study results showed that adverse events occurred in around $8 \%$ of the reviewed records, ranging from $2.5 \%$ to $18.4 \%$. Therapeutic errors were the origin of about $34 \%$ of these adverse events, followed by diagnostic and operative errors at $19.1 \%$ and $18.4 \%$, respectively (5). Another estimate suggested that the true figure could be twice as high (6).

Medical errors and patient harm are distinct in many ways for children compared to adults (7). Serious errors in pediatric care occur more often in critical care settings and adverse drug events occur three times more common among pediatric patients than among adults (8).

An incident reporting system (IRS) in patient safety is a tool to communicate safety-relevant information that allows clinicians to learn about and from patient safety incidents (9). IRSs are a method of error reporting, created to 
get information about patient safety that can then be interpreted into individual and organizational learning (10). Several studies have emphasized the implementation of reporting systems as an essential step in patient safety improvement $(11,12)$. The reported incidents should include not only sentinel events, but also small, everyday process failures that can be easily tackled (12).

For organizations to improve their safety performance, managers must be aware of incidents in their organizations and employees must feel assertive about reporting errors and near misses without the worry of recrimination (2). Voluntary confidential reporting deepens the understanding of the frequency of adverse events, near misses, and their trends and hence acts as a warning system (13).

Our study hypothesized that an educational training program for health care professionals on IRS would enhance their attitudes and practices on event reporting and would serve as a basis for improving patient safety in the future.

\section{Objectives}

The study aimed to enhance patient safety practices in pediatric intensive care units (PICUs) in a teaching hospital in Cairo through enhancing health care professionals' error reporting attitudes and practices.

\section{Methods}

\subsection{Study Design}

A quasi-experimental pretest-posttest study was conducted to determine the impact of a training program on attitudes and practice of event reporting among health care professionals before and after the intervention (14).

\subsection{Study Setting and Population}

The study was conducted from March to December 2018 at four PICUs of a teaching hospital in Cairo, Egypt. All four general PICUs of the hospital were included in the study. All the studied general PICUs had similar organizational and staffing structures. All physicians and nursing staff working in the four general PICUs who were available and consented to participate in the study at the time of research were included, as illustrated in Figure 1.

In-depth interviews (IDIs) were conducted with 16 health care providers, including 4 managers, 4 head nurses, 4 physicians, and 4 nurses each from an ICU. The IDIs aimed to reach a better understanding of the adverse event reporting situation in the PICUs and to assess the staff's attitude and readiness to implement an IRS. The interviews' questions were about the perceived challenges of interviewees concerning the IRS implementation and suggestions to overcome those difficulties.

A tailored education program was developed for health care professionals focusing on enhancing attitudes and practices of incident reporting. The program consisted of 120 minutes small group workshop sessions with approximately 6 to 8 participants, carried out by a member of the study team. In total, 12 sessions (three in each ICU), each for about 30 minutes and 15 minutes for open questions and discussion, respectively, were done over a period of eight weeks. The program started with a one-day face-to-face workshop, followed by hanging posters and handing out reporting formats to the staff at their workplace. Tools for the educational program included booklets containing the scientific material used in the presentations attended by the healthcare personnel and leaflets containing the patient safety goals in English and Arabic. The workshop consisted of a lecture, scenarios, and group discussion. Graphic posters on incident reporting were placed at the PICUs. An IRS was established in the PICUs of the teaching hospital. A labeled box was installed next to the "sign-in" station of the health-care personnel to encourage the voluntary, anonymous reporting by facilitating the process. Printed copies of the "incident reporting form" were placed in each of the ICU's and kept in an easily accessible place. The form was designed based on the "international conceptual patient safety (ICPS) framework" (15), with items on the following: Incident type, Patient characteristics, Contributing factors, Mitigating factors, Actions taken to reduce the risk, How the incident was discovered, Patient harmed or not and how. The questionnaire used to evaluate the intervention was adapted from the hospital survey on patient safety culture (HSOPSC) developed by the agency for health care research and quality (16). Questions that were relevant to the aim of our research were selected from the original questionnaire. The survey tool included nine items in two domains including communication (6 items) and frequency of error reporting (3 items). A section for demographic and background information was provided, including age, sex, ICU department, Job title, duration of working in the hospital, and average working hours. A five-point-Likert type scale was used to measure the staff's attitude, with possible responses ranging from 1 to 5 . A pilot testing phase was carried out to adapt the questionnaire and verify that its items and questions were comprehensible and clear. Data collection was undertaken inside the PICUs after presenting a brief introduction to healthcare providers about the aim of the questionnaire. The questionnaires were distributed while making sure that each healthcare provider answered the questions alone.

Health care providers were approached to participate 


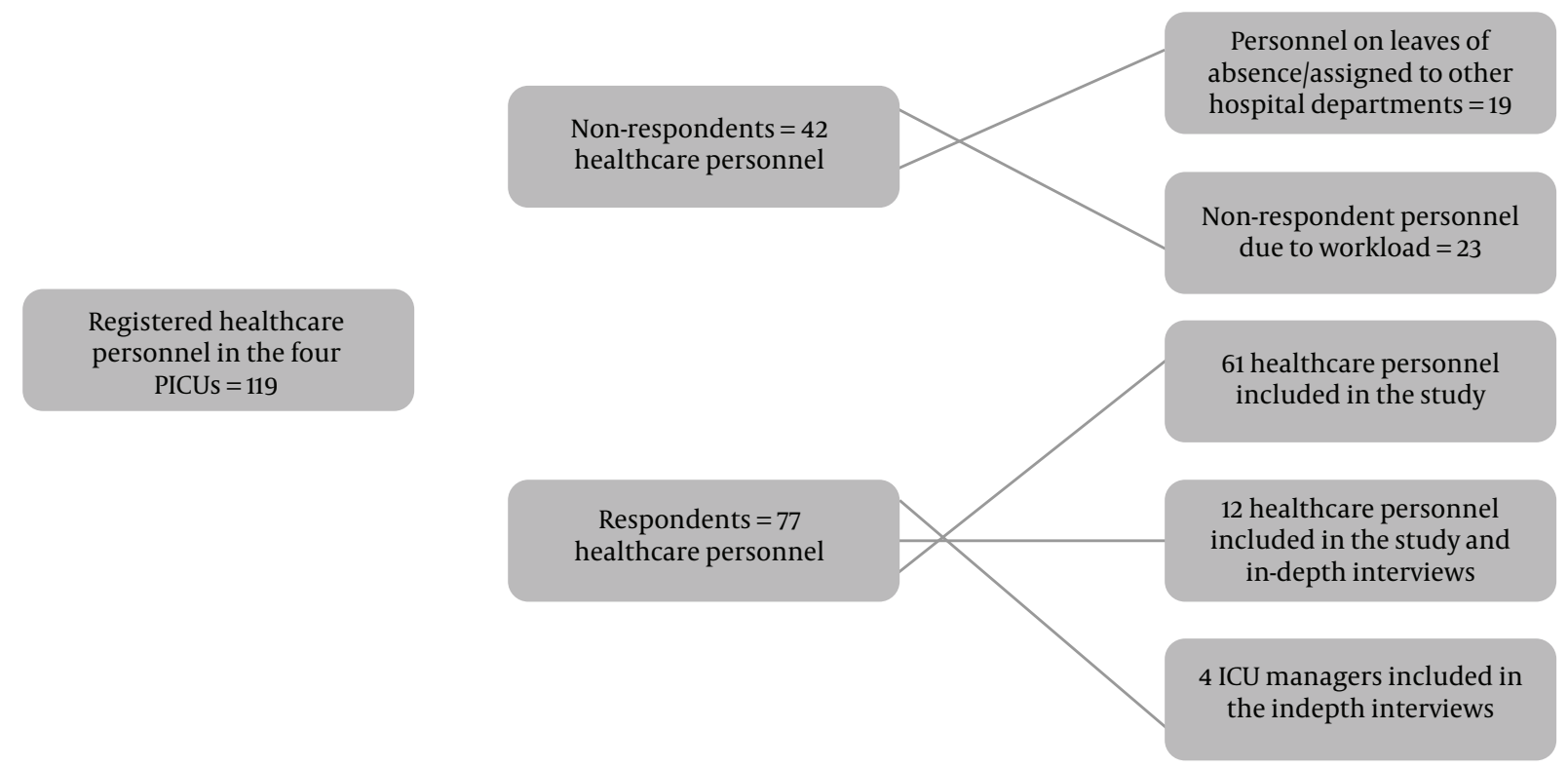

Figure 1. Flow diagram of healthcare personnel recruitment in the study

in the survey before and three months after the intervention. Eventually, 73 participants completed the survey. All surveys were anonymous and voluntary. The study was approved by the Cairo University Ethics Review Committee.

\subsection{Data Management and Statistical Analysis}

All collected data were revised for completeness and logical consistency. Pre-coded data were entered into a computer using a database developed for data entry on Microsoft Office Excel program for Windows 10. Data were then transferred to the computer program IBM SPSS (Statistical Package for the Social Science; IBM Corp, Armonk, NY, USA) release 22 for Microsoft Windows for data analysis. There has been a controversy about whether converting ordinal data of Likert scale to numbers can be treated as interval data. Means and standard deviations have unclear meanings when applied to Likert scale responses. Furthermore, response data on trainee's evaluation experiences are clustered at high and low extremes. Because of these observations, experts have contended that frequencies (percentages of responses in each category) or MannWhitney U test should be used for analysis instead of parametric tests (17). For this reason, grouping of responses to the incident reporting attitude questionnaire was undertaken in the current study to facilitate discussion and comparison with similar studies (18), as follows: Positive response (strongly agree and agree responses: scores 5 and 4 on the Likert scale), Neutral response (neither: score 3), and Negative response (strongly disagree and disagree re- sponses: scores 1 and 2 on the Likert scale). The direction of responses was reversed in negative wording questions. A composite score was calculated, which was the average percentage of positive responses of the survey items in each dimension (19). The reported error rate was calculated, as follows:

Number of reported errors $\times 100$

Total no. of admissions Data were tested for normality using the Kolmogorov-Smirnov test and the ShapiroWilk test. Descriptive statistics were used to summarize the data. Comparison between pre-and post-program data was made using the McNemar test for qualitative variables; the test was used to compare between positive responses and negative responses in the baseline assessment versus the follow-up assessment for each item in the attitude questionnaire in $2 \times 2$ tables. A comparison of numerical variables by the Mann-Whitney test and Kruskal Wallis test was performed to study the relationship between demographic and occupational characteristics and participants' responses.

Two-group tests can accommodate quasi-experimental data. In two-group (bivariate) tests, student $t$-tests are used for continuous outcomes. However, student $t$-tests are sensitive to outlying values. Therefore, a non-parametric test can be used (20). Pvalues of less than 0.05 were considered statistically significant. IDIs were semi-structured and consisted of open-ended questions, audiotaped face-to-face interviews of up to 30 minutes duration. The data analysis was carried out using inductive thematic analysis. 


\subsection{Ethical Consideration}

The study was approved by the Cairo University Ethics Review Committee (code No. 1209).

\section{Results}

The data of IDIs with 16 health care personnel were analyzed, organized, and presented according to the following key themes.

\subsection{Knowledge and Awareness of Incident Reporting}

The interviewed health care personnel acknowledged incident reporting as an essential component to improve learning and patient safety culture. However, they highlighted that no incident reporting system was yet established in the hospital and most hospital health care personnel did not report any events unless they were sentinel events. The majority of the interviewees $(n=12)$ thought that event reporting is the mere reporting of major errors that occur during the process of care of patients leading to their harms. They were not aware that it also relies on staff's reports of errors, safety concerns, adverse events, and near misses that occur within routine situations to learn from it.

\subsection{Barriers to Incident Reporting}

A manager said, "Incident reporting is perceived as if I am complaining someone, so it never happens unless it's a disaster and someone has to be punished".

A nurse said, "I remember a case who had major complications due to health care personnel mistakes but a young nurse was just scapegoated without proper investigation of the cause, and now it is forgotten without learning from it and it might be repeated in the future".

Another nurse mentioned, "We never report errors unless we have to and if it was a very obvious mistake that had caused harm to a patient; like once a glass of hot tea was dropped on the leg of a patient from a close table, and the nurse who caused that get fired, although she didn't mean it, and the table is still in the same place, close to the patient bed".

The error reporting attitude questionnaire was distributed to 96 health care personnel in the four studied units. The response rate for the pre-intervention questionnaire was 76\%. The post-intervention questionnaire was distributed to those who responded to the preintervention questionnaire and the post-intervention response rate was $100 \%$.

The results showed a relationship between demographic and occupational characteristics and attitudes towards error reporting among health care professionals at the baseline and follow-up phase (Tables 1 and 2). The impact of the training program on changes in attitudes is presented in Table 3 through a comparison between positive attitudes towards error reporting in the pre-intervention phase versus post-intervention. In Figure 2, the frequency of the practices of error reporting is illustrated to show the changes in practices in the post-intervention phase. Finally, the frequency of causes of errors is reported.

The median of the age of the study participants was 30 with IQR of 28 - 40. Table 1 describes the characteristics of the participants. About $70 \%$ of the health care personnel involved in the study were females. The majority of the included personnel were nurses $(n=45)$ representing $61.6 \%$ of the total personnel. More than half $(n=43)$ of the included personnel have been working in the hospital from one to 15 years before the study. The majority (95.8\%) stated that they worked 80 hours per week or more. On comparing the total scores of error reporting attitude at the baseline among females and males, no statistically significant difference was found, so was between various groups of working PICUs, the duration of work in the hospital, and job title.

Table 3 illustrates the positive change in communication openness, feedback about errors, and reporting attitude among health care personnel. The average composite score increased significantly for the "Frequency of error reporting" dimension from $23.3 \%$ to $42 \%$. The percentage of health care personnel who felt free to report anything that might negatively affect the patient care increased from $46.6 \%$ before the intervention to $69.9 \%$ after the intervention ( $\mathrm{P} \leq 0.001)$. Moreover, $90.4 \%$ of the health care personnel discussed the ways to prevent errors in the followup assessment versus 79.5\% in the baseline assessment $(\mathrm{P}=$ 0.001). The frequency of reporting corrected errors and errors that could not do any harm to patients increased significantly from $24.7 \%$ in the baseline assessment to $38.4 \%$ and $46.6 \%$, respectively, in the follow-up phase.

of the 918 patients admitted to the four study units in the post-intervention phase, 215 potential errors were reported during the following six months. It started with 34 errors per 100 admissions reported during the first month and ended with 16 errors per 100 admissions reported in the sixth month (Figure 2).

The problems associated with the reported errors were as follows. Communication problems between health care personnel accounted for $34 \%$ of the potential errors reported. The majority of the communication errors were regarding the lack of communication between nurses and doctors, particularly in telephone orders and verbal orders. Hygiene errors accounted for $32 \%$, by reporting the non-adherence of some health care personnel to hand hygiene guidelines supposedly established in the PICUs, particularly by doctors, who were too busy to attend the 


\begin{tabular}{|c|c|c|c|}
\hline Item & $\mathrm{N}=\mathbf{7 3}$, No. $(\%)$ & Baseline Attitude Score, Median (IQR) & P Value \\
\hline Gender & & & $0.4^{\mathrm{a}}$ \\
\hline Male & $22(30.1)$ & $2.9(2.6-3.1)$ & \\
\hline Female & $51(69.9)$ & $2.9(2.4-3.1)$ & \\
\hline ICU & & & $0.7^{\mathrm{b}}$ \\
\hline ICU A & $18(24.7)$ & $2.7(2.4-3.1)$ & \\
\hline ICU B & $18(24.7)$ & $2.8(2.6-3.00)$ & \\
\hline ICU C & $18(24.7)$ & $2.8(2.4-3.1)$ & \\
\hline ICU D & $19(26.0)$ & $3.0(2.6-3.2)$ & \\
\hline Duration of work in the hospital, $y$ & & & $0.06^{\mathrm{b}}$ \\
\hline$<1$ & $4(5.5)$ & $2.8(2.4-3.00)$ & \\
\hline $1-5$ & $8(11)$ & $3.1(2.6-3.4)$ & \\
\hline $6-10$ & $18(24.7)$ & $3.3(2.5-3.6)$ & \\
\hline $11-15$ & $43(58.9)$ & $2.7(2.4-2.9)$ & \\
\hline$>15$ & $21(28.8)$ & $2.7(2.6-3.1)$ & \\
\hline Job title & & & $0.06^{\mathrm{b}}$ \\
\hline Staff physician & $43(58.9)$ & $2.7(2.2-3.1)$ & \\
\hline Head nurse & $28(38.4)$ & $2.5(2.2-2.8)$ & \\
\hline Resident physician & $15(20.5)$ & $2.8(2.6-3.0)$ & \\
\hline Nurse & $9(12.3)$ & $3.0(2.6-3.3)$ & \\
\hline Average weekly working hours, h & & & $0.4^{\mathrm{b}}$ \\
\hline $20-39$ & $1(1.4)$ & $3.2(3.1-3.3)$ & \\
\hline $40-79$ & $2(2.7)$ & $2.7(2.6-3.1)$ & \\
\hline$\geq 80$ & $70(95.8)$ & $2.9(2.4-3.1)$ & \\
\hline
\end{tabular}

${ }^{\mathrm{a}}$ Mann-Whitney test.

${ }^{\mathrm{b}}$ Kruskal-Wallis test.

hand hygiene program sessions implemented in the PICUs. These were followed by therapeutic error reports, accounting for $29 \%$. The most common therapeutic errors were related to medications (18\%) whether wrong dose, route, or time in addition to proper medications' admixing with proper solutions, followed by process problems involving the delivery of clinical and support services with $11 \%$ of the reports. Diagnostic problems accounted for $5 \%$ of the reports, where the most common ones were missed infections.

Table 2 shows the relationship between demographic and occupational characteristics and follow-up total attitude score. No statistically significant differences were found in the follow-up attitude score.

\section{Discussion}

The study evaluated the effectiveness of a face-to-face educational intervention in enhancing the incident re- porting practice of health care providers in PICUs of teaching hospitals in Cairo, Egypt. The results suggested that the program improved the incident reporting culture.

Qualitative research has always been believed to aid quantitative research in exploring and understanding the study situation in a deeper way and from different perspectives. Incident reporting is extremely crucial, particularly to increase health care quality. Similar to other studies (21, 22 ), the current study conducted qualitative in-depth interviews and focus-group discussions to improve acceptance and awareness of the reporting system by further exploring the reasons behind the personnel fear of reporting.

The diversity of interviewees may have ensured that a range of perspectives was captured successfully. The majority of the interviewees in the current study stated that they liked the idea of having an anonymous reporting system. This is similar to the findings of an Ethiopian study (22), where the interviewees showed a positive attitude 


\begin{tabular}{|c|c|c|}
\hline Item & Follow-up Attitude Score, Median (IQR) & P Value \\
\hline Gender & & $0.49^{\mathrm{a}}$ \\
\hline Male & $2.6(2.6-2.8)$ & \\
\hline Female & $2.7(2.2-3.0)$ & \\
\hline ICU & & $0.16^{\mathrm{b}}$ \\
\hline ICU A & $2.6(2.4-3.0)$ & \\
\hline ICU B & $2.7(2.8-3.1)$ & \\
\hline ICU C & $2.4(2.2-3.0)$ & \\
\hline ICU D & $2.7(2.7-3.0)$ & \\
\hline Duration of work in the hospital, $y$ & & $0.07^{\mathrm{b}}$ \\
\hline$<1$ & $2.8(2.6-3.00)$ & \\
\hline $1-5$ & $2.9(2.2-2.5)$ & \\
\hline $6-10$ & $2.7(2.6-2.9)$ & \\
\hline $11-15$ & $2.4(2.4-2.6)$ & \\
\hline$>15$ & $2.4(2.2-2.5)$ & \\
\hline Job title & & $0.37^{\mathrm{b}}$ \\
\hline Staff physician & $2.8(2.8-3.1)$ & \\
\hline Head nurse & $2.7(2.8-3.0)$ & \\
\hline Resident physician & $2.9(1.9-3.1)$ & \\
\hline Nurse & $3.0(2.4-3.2)$ & \\
\hline Average weekly working hours, h & & $0.39^{\mathrm{b}}$ \\
\hline $20-39$ & $3.2(3.2-3.3)$ & \\
\hline $40-79$ & $2.8(2.6-3.0)$ & \\
\hline$\geq 80$ & $2.8(2.8-3.0)$ & \\
\hline
\end{tabular}

${ }^{\text {a } M a n n-W h i t n e y ~ t e s t . ~}$

${ }^{\mathrm{b}}$ Kruskal Wallis test.

toward incident reporting and communication openness among health care professionals.

Average positive responses in the domains "Communication openness" and "Communication and feedback about errors" were found to be $47.6 \%$ and $49.8 \%$, respectively, in the baseline assessment. Aboul-Fotouh et al. in 2012 (19) found the corresponding rates to be 35\% and $39.7 \%$, respectively. The study findings showed that items of communication openness and communication and feedback about errors improved after the program intervention. There was a positive change in all items with a significant improvement in the attitude of the staff toward speaking up if something may affect negatively the patient care, discussing ways to prevent errors, and asking questions when something is not right. These findings were in line with the findings of a study (23) by Andreoli et al. in 2010. However, they were in contrast to the results of another study in which patient safety education of nurses and hos- pital staff did not improve their attitudes on communication openness (24). Be that as it may, the current study had another aspect in its intervention, which was teaching the health care personnel to speak up through incident reporting, especially about near misses; this may explain the differences between the current findings and those from previous studies.

Incident reporting in a vulnerable environment such as a PICU is considered extremely vital for building a patient safety climate. Our study mimicked other local incident reporting systems in hospitals, which typically used an incident reporting form comprising basic clinical details and a brief description of the incident (25). Baseline positive responses in the domain of frequency of event reporting were 23.8\%. Aboul-Fotouh et al. (19) reported higher rates (33\%) in the same domain. The frequency of incident reporting in our study increased significantly in the follow-up assessment to $42 \%$. These results mirror the find- 


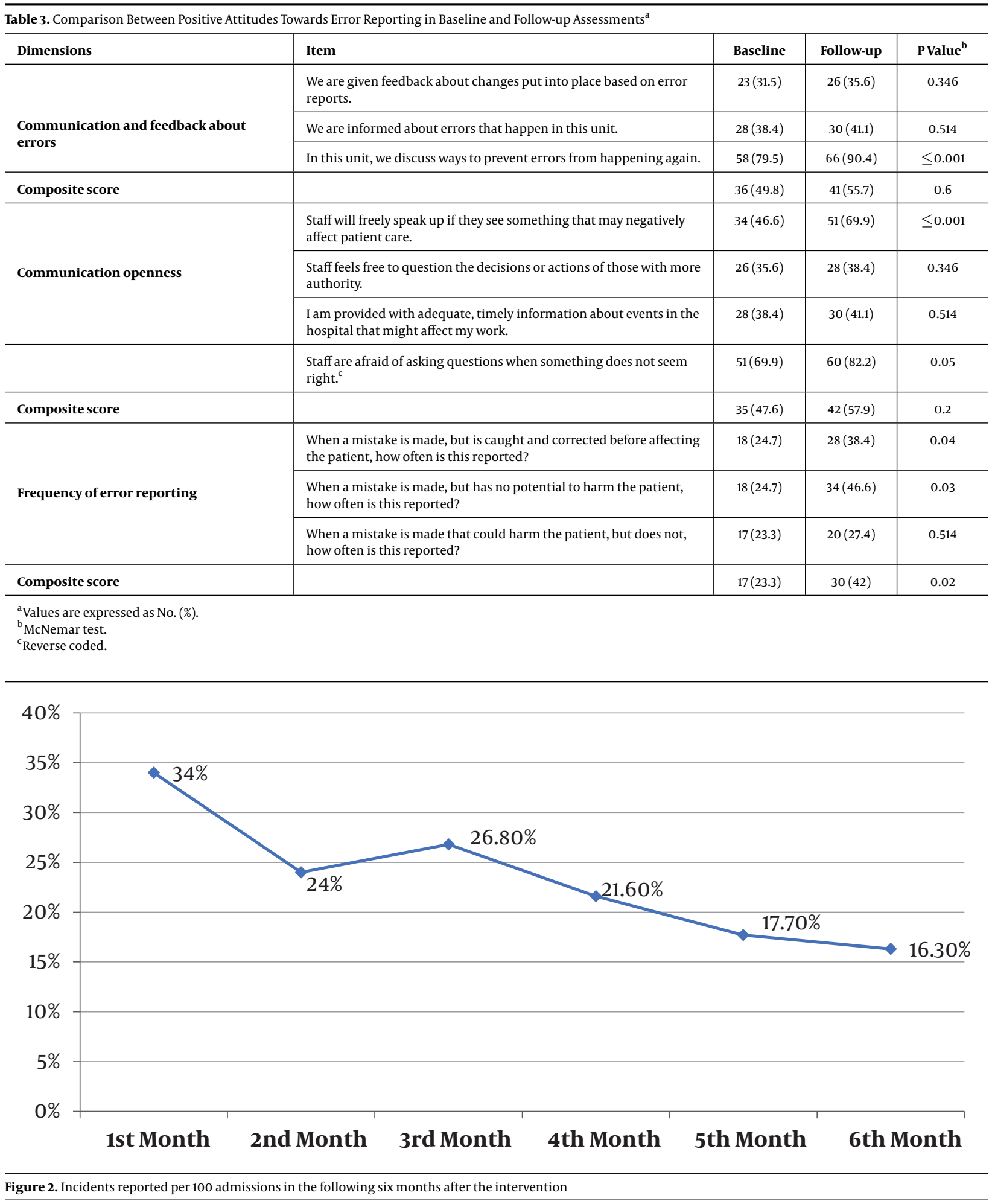

ings of other studies that have highlighted that education could improve the way in which health care professionals perceive errors (26).

Consequently, based on the current results, we can con- 
clude that education can improve error reporting. However, the incident reporting rate decreased from 34 per 100 admissions in the first month after the intervention to $16 \%$ in the sixth month in the follow-up phase. In a study (27) in the US, where 63 participants were included in an education program for IRS implementation, the numbers of events reported over the period of six months were 10 in the first month, 6 in the second month, 10 in the third month, 18 in the fourth month, 11 in the fifth month, and 6 in the sixth month. Which in total might be less than in the current study but given the different sites of the studies, as it wasn't in a PICU with all its complex health care disciplines, but was on a whole hospital, with its various departments, whether critical care or not.

In the same study (27), the results of qualitative analysis of the residents' interviews after patient safety education showed that they mentioned that they had found it quite difficult to carry out the course content in practice, the information learned faded away after the course, and it would be helpful to organize refresher courses. In addition, barriers related to behavioral control like high work-pressure might be effective (28). In the current study, more than $95 \%$ of the participants mentioned working $\geq$ 80 hours per week. The barriers are hard to overcome by educating health care personnel only. They could be addressed through adjusting policies, training the context of the health care personnel to create a generation culture in which safety is better perceived at all culture levels, and providing the required resources to monitor and analyze the causes of errors $(28,29)$.

In a study to create a physician-based voluntary incident reporting system for adverse events and medical errors (30), the frequency of problematic processes of care associated with reported potential errors was as follows. Therapeutic errors including medication errors accounted for $33.3 \%$ of the potential adverse events while $37.3 \%$ were due to problems involving the delivery of clinical and support services. Diagnostic problems accounted for about $19 \%$ of reports. Moreover, $2.1 \%$ of potential errors were due to poor communication. In our study, poor communication was responsible for more than one-third of potential errors (34\%) while $29 \%$ and $5 \%$ of reported errors were due to therapeutic and diagnostic problems, respectively.

The study has prominent strengths. This is the first study to evaluate the effect of an intervention to create a voluntary anonymous IRS in PICUs in a teaching hospital in Cairo. The IRS enhanced the capture of near-miss events due to the anonymity and/or ease of the reporting process, as well as providing a format to report these events. Knowledge of near-miss may lead to the development of systems to improve the care of critically ill pediatric patients. The survey tool was adapted from a validated questionnaire.

One of the most important factors to help enhance the incident and error reporting is to overcome the fear of punishment among the personnel. This was done in our study by involving the hospital management at all levels to support and reassure the healthcare personnel about the importance of incident reporting for them, for patients, and ultimately for the hospital quality of care. In addition, representatives from hospital management attended the educational sessions to encourage them and help them use the incident reporting system.

However, there were some limitations. One-group pretest-posttest design was used with its threats to validity. Attending workshops voluntarily might have led to selection bias. Analysis of qualitative data may have been subjected to interpretation bias. Hence, an incident reporting attitude questionnaire was used. A short survey tool was designed to encourage participation and completion by health care personnel. The effect of education was studied only once, i.e., 3 months after the intervention. Future research is needed to examine the sustainability of health care positive attitudes.

It was a totally novel idea to implement an incident reporting system with the enhancement of overall safety culture. Thus, the change in important errors was not investigated. However, we plan to perform a follow-up study involving important errors and their reporting and how to manage them.

\subsection{Conclusions}

A face-to-face educational intervention was effective in changing the attitude toward incident reporting among health care professionals. The frequency of incident reporting improved significantly after the intervention. Barriers related to the health care professional-organizational context need to be addressed.

\section{Footnotes}

Authors' Contribution: John Rene Labib: study concept and design, interpretation of data, and critical revision of the manuscript. Nesreen Mohamed Kamal Elden: Study concept and design, analysis and interpretation of data, and drafting of the manuscript. Amira Aly Hegazy: study concept and design, acquisition of data, and analysis and interpretation of data. Nargis Albert Labib: study concept and design, study supervision, and critical revision of the manuscript.

Conflict of Interests: The authors declare that they have no competing interests.

Ethical Approval: This study was conducted as a research study at the Cairo University Teaching Hospital and approved by the Ethics Review Committee of the Public Health Department, Cairo University, and received the code of ethics S12019. 
Funding/Support: This study was supported by the Cairo University Teaching Hospital.

\section{References}

1. Wilson R, Giberd R, Michel P. WHO EMRO-AFRO adverse event prevalence study aggregated results for 8 countries. Sydney, Australia: Northern Center for Health Care Improvement; 2008. Available from: https:// www.who.int/patientsafety/topics/research/emro-afro-studies/en/.

2. Hudson P. Applying the lessons of high risk industries to health care. Qual Saf Health Care. 2003;12 Suppl 1:i7-12. doi: 10.1136/qhc.12.suppl_1.i7. [PubMed: 14645741]. [PubMed Central: PMC1765769].

3. World Health Organization. World Health Organization (2010): Patient safety. 2018. Available from: http://www.euro.who.int/en/what-wedo/health-topics/Health-systems/patient-safety.

4. de Vries EN, Ramrattan MA, Smorenburg SM, Gouma DJ, Boermeester MA. The incidence and nature of in-hospital adverse events: A systematic review. Qual Saf Health Care. 2008;17(3):216-23. doi: 10.1136/qshc.2007.023622. [PubMed: 18519629]. [PubMed Central: PMC2569153].

5. Wilson RM, Michel P, Olsen S, Gibberd RW, Vincent C, El-Assady R, et al. Patient safety in developing countries: Retrospective estimation of scale and nature of harm to patients in hospital. BMJ. 2012;344. e832. doi: 10.1136/bmj.e832. [PubMed: 22416061].

6. James JT. A new, evidence-based estimate of patient harms associated with hospital care. J Patient Saf. 2013;9(3):122-8. doi: 10.1097/PTS.ob013e3182948a69. [PubMed: 23860193].

7. Santell JP, Hicks R. Medication errors involving pediatric patients. Jt Comm J Qual Patient Saf. 2005;31(6):348-53. doi: 10.1016/s15537250(05)31046-4. [PubMed: 15999965].

8. Suresh G, Horbar JD, Plsek P, Gray J, Edwards WH, Shiono PH, et al. Voluntary anonymous reporting of medical errors for neonatal intensive care. Pediatrics. 2004;113(6):1609-18. doi: 10.1542/peds.113.6.1609. [PubMed: 15173481].

9. Vincent C. Reporting and learning systems. Patient safety. 2nd ed. Chichester: Wiley-Blackwell; 2010. p. 75-95. doi: 10.1002/9781444323856.ch5.

10. Currie G, Waring J, Finn R. The limits of knowledge management for Uk public services modernization: The case of patient safety and service quality. Public Adm. 2008;86(2):363-85. doi: 10.1111/j.14679299.2007.00705.x.

11. Gambone JC, Reiter RC. Elements of a successful quality improvement and patient safety program in obstetrics and gynecology. Obstet Gynecol Clin North Am. 2008;35(1):129-45. x. doi:10.1016/j.ogc.2007.12.004. [PubMed: 18319132].

12. Mirabello J. Getting to havarti: Moving toward patient safety in obstetrics. Obstet Gynecol. 2008;111(3):777. author reply 777-8. doi: 10.1097/AOG.0b013e318166e86f. [PubMed: 18310386].

13. Dopfer K, Foster J, Potts J. Micro-meso-macro. J Evol Econ. 2004;14(3):263-79. doi: 10.1007/s00191-004-0193-0.

14. Harris AD, McGregor JC, Perencevich EN, Furuno JP, Zhu J, Peterson $\mathrm{DE}$, et al. The use and interpretation of quasi-experimental studies in medical informatics. J Am Med Inform Assoc. 2006;13(1):1623. doi: 10.1197/jamia.M1749. [PubMed: 16221933]. [PubMed Central: PMC1380192].

15. World Health Organization. International classification of patient safety (WHO-ICPS): World Health Organization, 2009. WHO patient safety research. 2018. Available from: www.who.int/patientsafety/taxonomy/ icps_full_report.pdf.
16. Sorra JS, Nieva VF. Hospital survey on patient safety culture. Rockville: MD: Agency for health care research and quality; 2004. p. 4-41.

17. Sullivan GM, Artino AR Jr. Analyzing and interpreting data from likerttype scales. J Grad Med Educ. 2013;5(4):541-2. doi: 10.4300/JGME-5-4-18. [PubMed: 24454995]. [PubMed Central: PMC3886444].

18. Elsous A, Akbarisari A, Rashidian A, Aljeesh Y, Radwan M, Abu Zaydeh H. Psychometric properties of an arabic safety attitude questionnaire (Short Form 2006). Oman Med J. 2017;32(2):115-23. doi: 10.5001/omj.2017.21. [PubMed: 28439381]. [PubMed Central: PMC5397085].

19. Aboul-Fotouh AM, Ismail NA, Ez Elarab HS, Wassif GO. Assessment of patient safety culture among healthcare providers at a teaching hospital in Cairo, Egypt. East Mediterr Health J. 2012;18(4):372-7. [PubMed: 22768700].

20. Shardell M, Harris AD, El-Kamary SS, Furuno JP, Miller RR, Perencevich EN. Statistical analysis and application of quasi experiments to antimicrobial resistance intervention studies. Clin Infect Dis. 2007;45(7):901-7. doi: 10.1086/521255. [PubMed: 17806059].

21. Anderson JE, Kodate N, Walters R, Dodds A. Can incident reporting improve safety? Healthcare practitioners' views of the effectiveness of incident reporting. Int J Qual Health Care. 2013;25(2):141-50. doi: 10.1093/intqhc/mzs081. [PubMed: 23335058].

22. Wami SD, Demssie AF, Wassie MM, Ahmed AN. Patient safety culture and associated factors: A quantitative and qualitative study of healthcare workers' view in Jimma zone Hospitals, Southwest Ethiopia. BMC Health Serv Res. 2016;16:495. doi: 10.1186/s12913-016-1757-z. [PubMed: 27644960]. [PubMed Central: PMC5029028].

23. Andreoli A, Fancott C, Velji K, Baker GR, Solway S, Aimone E, et al. Using SBAR to communicate falls risk and management in interprofessional rehabilitation teams. Healthc Q. 2010;13 Spec No:94-101. [PubMed: 20959737].

24. AbuAlRub RF, Abu Alhijaa EH. The impact of educational interventions on enhancing perceptions of patient safety culture among Jordanian senior nurses. Nurs Forum. 2014;49(2):139-50. doi: 10.1111/nuf.12067. [PubMed: 24392690].

25. Vincent C. Incident reporting and patient safety. BMJ. 2007;334(7584):51. doi: 10.1136/bmj.39071.441609.80. [PubMed: 17218667]. [PubMed Central: PMC1767279].

26. Nie Y, Li L, Duan Y, Chen P, Barraclough BH, Zhang M, et al. Patient safety education for undergraduate medical students: A systematic review. BMC Med Educ. 2011;11:33. doi: 10.1186/1472-6920-11-33. [PubMed: 21669007]. [PubMed Central: PMC3128569].

27. Krouss M, Alshaikh J, Croft L, Morgan DJ. Improving incident reporting among physician trainees. J Patient Saf. 2016. doi: 10.1097/PTS.0000000000000325. [PubMed: 27617963]. [PubMed Central: PMC5344764].

28. Jansma JD, Wagner C, Bijnen AB. Residents' intentions and actions after patient safety education. BMC Health Serv Res. 2010;10:350. doi: 10.1186/1472-6963-10-350. [PubMed: 21194435]. [PubMed Central: PMC3224360].

29. Elden NM, Ismail A. The importance of medication errors reporting in improving the quality of clinical care services. Glob J Health Sci. 2016;8(8):54510. doi: 10.5539/gjhs.v8n8p243. [PubMed: 27045415]. [PubMed Central: PMC5016354 publication of this paper].

30. Weingart SN, Callanan LD, Ship AN, Aronson MD. A physicianbased voluntary reporting system for adverse events and medical errors. J Gen Intern Med. 2001;16(12):809-14. doi: 10.1046/j.15251497.2001.10231.x. [PubMed: 11903759]. [PubMed Central: PMC1495298]. 